\title{
Educação em saúde com adolescentes escolares acerca da sexualidade e infecções sexualmente transmissíveis: um relato de experiência
}

\author{
Health education with school adolescents about sexuality and sexually transmitted \\ infection: an experience report
}

\section{Educación en salud con adolescentes escolares acerca de la sexualidade e infeccciones sexualmente transmisibles: uma cuenta de experiencia}

Juliana de Castro Nunes Pereira ${ }^{1 *}$, Luciana Uchôa Barbosa ${ }^{1}$, Amanda Haissa Barros Henriques², Patrícia Maria de Oliveira Andrade Araújo ${ }^{3}$, Marcela Lourene Correia Muniz ${ }^{3}$, Edja Clébya dos Santos Melo ${ }^{4}$, Andrezza Renata Araujo de Figuerêdo Priori ${ }^{5}$.

\section{RESUMO}

Objetivo: Descrever a experiência de um projeto de extensão com adolescentes escolares sobre sexualidade, Infecções Sexualmente Transmissíveis e HIV/AIDS. Relato de experiência: Estudo descritivo, do tipo relato de experiência, desenvolvido por discentes e docentes do Curso Técnico de Enfermagem do Instituto Federal de Pernambuco Campus Belo Jardim. O público alvo englobou alunos do 1ํano do Ensino Médio - Integrado dos Cursos Técnicos de Informática, Agropecuária e Agroindústria. Durante as ações foram realizadas palestras, exibição de vídeos, teatros. Os alunos puderam compartilhar experiências e esclarecerem dúvidas acerca da sua sexualidade, bem como sobre a prevenção da gravidez e ISTs, favorecendo a ampliação de conhecimentos sobre as temáticas em questão, de maneira participativa. Considerações finais: Evidenciou-se a importância de atividades educativas na adolescência que tenham como base as temáticas trabalhadas, visando a promoção da saúde por meio de métodos que favoreçam a prática da sexualidade de maneira segura e saudável.

Palavras-chave: Educação em saúde, Adolescente, Doenças sexualmente transmissíveis, Sexualidade.

\begin{abstract}
Objective: To describe the experience lived during an extension project developed with school adolescents and emphasizes questions about sexuality, Sexually Transmissible Infections and HIV/AIDS. Experience report: This is a descriptive research based on an experience report, developed by students and teachers of the Nursing Technical Course of the Federal Institute of Pernambuco Campus Belo Jardim. The activities were developed with students the 1st year of High School - Integrated Technical Courses in Informatics, Agriculture and Agroindustry. The actions consisted in the presentation of lectures, videos and plays. The students were able to share experiences and clarify doubts about their sexuality, as well as the prevention of

\footnotetext{
1 Instituto Federal de Educação, Ciência e Tecnologia de Pernambuco (IFPE), campus Belo Jardim, Belo Jardim - Pernambuco. *E-mail: juli_decastro@hotmail.com

${ }^{2}$ Instituto Federal de Educação, Ciência e Tecnologia da Paraíba (IFPB), campus Mangabeira, João Pessoa

- Paraíba.

${ }^{3}$ Instituto Federal de Educação, Ciência e Tecnologia de Pernambuco (IFPE), campus Abreu e Lima, Abreu e Lima - Pernambuco.

${ }^{4}$ Secretaria Municipal de Saúde de Belo Jardim, Belo Jardim - Pernambuco.

${ }^{5}$ Autarquia Educacional de Belo Jardim, Faculdade do Belo Jardim, Belo Jardim - Pernambuco.
}

SUBMETIDO EM: 6/2019

ACEITO EM: 7/2019

PUBLICADO EM: 8/2019 
pregnancy and STIs, favoring the expansion of knowledge about the subject matter in a participatory manner. Final considerations: It was evidenced the importance of educational activities in adolescence based on the themes worked, aiming at promoting health through methods that favor the practice of sexuality in a safe and healthy way.

Keywords: Health education, Teenager, Sexually transmitted diseases.

\section{RESUMEN}

Objetivo: Describir la esperiencia de um proyecto de extensión con adolescentes escolares sobre sexualidad, Infecciones Sexualmente Transmisibles y HIV/SIDA. Relato de experiencia: Estudio descriptivo, del tipo relato de experiencia, desarrollado por discentes y docentes del Curso Técnico de Enfermería del Instituto Federal de Pernambuco Campus Belo Jardim. El público objetivo englobó estudiantes del $1^{\circ}$ año de la escuela secundaria - Integrado de los Cursos Técnicos de Informática, Agropecuaria y Agroindustria. Durante las acciones se realizaron conferencias, exhibición de vídeos, teatros. Los estudiantes pudieron compartir experiencias y aclarar dudas acerca de su sexualidad, así como sobre la prevención del embarazo e IST's, favoreciendo la ampliación de conocimientos sobre las temáticas em cuestión de manera participativa. Consideraciones finales: Se evidenció la importancia de actividades educativas en a adolescencia que tengan como base las temáticas trabajadas, visando la promoción de la salud por medio de métodos que favorezcan la práctica de la sexualidad de manera segura y saludable.

Palabras-clave: Educación en salud, Adolescente, Enfermedades de transmisión sexual.

\section{INTRODUÇÃO}

A adolescência é um momento decisivo no desenvolvimento sexual humano, pois é nela que a sexualidade se generaliza, ocorrendo intensas transformações biopsicossociais. O Ministério da Saúde segue a convenção elaborada pela Organização Mundial da Saúde (OMS) que delimita o período entre 10 e 19 anos, 11 meses e 29 dias de idade como adolescência, e o situado entre 15 e 24 anos como juventude. Há portanto, uma interseção entre a segunda metade da adolescência e os primeiros anos da juventude (BRASIL, 2010).

A descoberta do prazer, muitas vezes, dá-se nessa época, onde por sua vez a sexualidade precoce aumenta a vulnerabilidade às Infecções Sexualmente Transmissíveis (IST), inclusive a contaminação pelo Vírus da Imunodeficiência Adquirida (HIV) e o desenvolvimento da Síndrome da Imunodeficiência Adquirida (AIDS), a gravidez na adolescência de forma não planejada e outros riscos, o que interfere em suas metas de vida futura (CARNEIRO RF, et al., 2015).

Apesar de existirem outras formas de infecção por HIV, a transmissão via relações sexuais penetrativas constitui ainda o principal meio de adquirir o vírus (OLIVEIRA LP e PATEL BN, 2015). A falta de conhecimento sobre o uso de um método preventivo deixa o jovem exposto a riscos. Desta forma, faz-se necessário adotar medidas de conscientização e elaboração de planos de educação em saúde para abordar o jovem e esclarecer sobre os riscos.

No entanto, a sexualidade entre os adolescentes ainda continua sendo um assunto que pode provocar polêmica na sociedade. Em algumas famílias, sexo é um assunto por vezes proibido de ser mencionado fazendo com que o jovem adolescente busque informações fora de casa. Estas informações podem estar erradas ou então, o adolescente deixa de tirar suas dúvidas por sentir-se inseguro ou constrangido em abordar este tema com uma pessoa que não é de seu convívio diário (KRABBE EC, et al., 2016).

Neste contexto, as ações de educação em saúde abordando temas como sexualidade, métodos contraceptivos e as IST's, HIV e AIDS são consideradas como ferramenta importante na promoção da saúde e prevenção de doenças. Segundo Amoras BC et al. (2015) é necessário construir um conhecimento 
crítico dos adolescentes em relação a sua saúde, favorecendo a eles o empoderamento de cuidar de si próprio, os responsabilizando de manter sua saúde e se prevenir contra as IST's e uma gravidez indesejada.

Nesta perspectiva, o Ministério da Educação por meio dos Parâmetros Curriculares Nacionais (PCN), incluiu, em 1997 a educação sexual entre os temas transversais nas diversas áreas do conhecimento, com finalidade de impregnar toda a prática educativa com as questões da orientação sexual (BIANCO AP, 2017).

Segundo Silva RAR, et al. (2016) a escola é um ambiente social no qual o indivíduo passa grande parte de sua vida, e é um dos principais elementos para contatos interpessoais, por isso deve contribuir para 0 desenvolvimento de uma educação sexual que promova no adolescente senso de autorresponsabilidade e compromisso para com a sua própria sexualidade.

Em face do exposto, objetivou-se com esta pesquisa descrever a experiência da realização de um projeto de extensão intitulado 'Educação em Saúde com Jovens Escolares: sexualidade, IST's, HIVIAIDS', oportunizando o acesso à educação sexual em seu próprio ambiente escolar, trazendo esclarecimento da prática sexual, das mudanças corporais, do uso correto de métodos contraceptivos e das IST's.

\section{MÉTODOS}

Trata-se de um estudo descritivo, do tipo relato de experiência, desenvolvido pelo Projeto de Extensão 'Educação em Saúde com Jovens Escolares: sexualidade, IST's, HIVIAIDS', o qual fora executado por discentes e docentes do Curso Técnico em Enfermagem do Instituto Federal de Educação, Ciência e Tecnologia do Pernambuco (IFPE), campus Belo Jardim-PE.

O projeto de extensão vivenciado de fevereiro a dezembro de 2016 foi submetido e aprovado pelo Comitê de Ética em Pesquisa da Autarquia Educacional do Belo Jardim -AEB, obtendo o Certificado de Apresentação para Apreciação Ética (CAAE) № 54659516.0.0000.5189 e Parecer de aprovação: 3.365.290.

As ações foram desenvolvidas mensalmente no IFPE, campus Belo Jardim-PE, município situado à 172 $\mathrm{Km}$ da capital Recife-PE, no agreste meridional Pernambucano. O público alvo foram alunos do $1^{\circ}$ ano do Ensino Médio - na modalidade de ensino Integrado, os quais cursam paralelamente ao Ensino Médio, os cursos técnicos em Agropecuária, Agroindústria e Informática. Participaram das ações extensionista 150 alunos, onde destes, $60 \%$ eram do sexo masculino e $40 \%$ do sexo feminino.

O projeto foi dividido em etapas; Etapa 1: elaboração do cronograma, subdivididos em temas específicos: Alterações fisiológicas e emocionais na adolescência; Métodos contraceptivos; Infecções Sexualmente Transmissíveis - IST's; Gravidez na Adolescência; Vírus da Imunodeficiência Humana AIDS/HIV. Etapa 2: contato com os adolescentes escolares para apresentação da proposta e assinatura do Termo de Consentimento Livre Esclarecido (TCLE). Etapa 3: planejamento e elaboração das ações educativas. Etapa 4: execusão das atividades com os adolescentes. E por fim a Etapa 5: avaliação e registro dos resultados das ações junto a equipe executora.

Durante cada ação foram elaboradas atividades específicas que incluíam vídeo educativo, aula expositiva, teatro simulando situações realísticas vivenciadas pelos adolescentes, dinâmica de perguntas e respostas, entre outras atividades.

Na temática específica de AIDS/HIV foi realizada uma parceria com a Secretaria Municipal de Saúde (SMS) de Belo Jardim-PE, onde profissionais da área de saúde levaram informações, sanando dúvidas sobre prevenção, sintomas e tratamentos das principais IST's, com ênfase na AIDS, realizando, inclusive, testes rápidos de HIV e Sífilis.

À medida que se realizavam as reuniões de planejamento das ações educativas, utilizava-se a técnica de observação e a descrição de todas as atividades desenvolvidas em um diário de campo.

Tais registros culminaram na elaboração do relatório final do Projeto de Extensão, assim como na construção deste artigo. 


\section{RESULTADOS E DISCUSSÃO}

As ações do Projeto de Extensão aconteceram no auditório principal do IFPE - campus Belo Jardim visando a melhor acomodação do público-alvo. A princípio houve uma apresentação da equipe, bem como exposição do Projeto de Extensão e seu objetivo. Durante o período das ações foram realizados 06 encontros com os adolescentes escolares. O primeiro encontro ocorreu para esclarecimento do projeto e essinatura do TCLE e os demais referente as ações educativas propriamente ditas.

A primeira ação educativa abordou o tema: Alterações fisiológicas e emocionais na adolescência. Para dar início a introdução da temática houve uma apresentação de uma palestra e exposição de vídeo voltado para 0 assunto. Durante a palestra alguns discentes extensionistas circularam no auditório com uma caixinha recolhendo perguntas anônimas, escritas em formulários entregues a cada um deles na chegada ao auditório. Ao final, foram sorteadas perguntas para que os participantes respondessem, seguida de explicação de forma dinâmica da equipe executora. Ao final da competição, os participantes que pontuassem mais nas respostas corretas seriam contemplados com um brinde. $O$ objetivo desta ação foi alcançado à medida que foram esclarecidas as dúvidas que os participantes do projeto tinham a respeito das principais alterações fisiológicas e emocionais da adolescência, possibilitando a eles maior conhecimento de seus corpos.

Nesta primeira ação os participantes puderam entender que as mudanças físicas, geralmente, vêm acompanhadas de alterações de humor, instabilidade emocional e muitos questionamentos e conflitos, que refletem no modo de pensar, sentir e se comportar.

As principais alterações fisiológicas elencadas pelos participantes foram: nos Meninos - surgimento de pelos nas regiões axilares, inguinais (pubianos), torácicos (peito) e faciais (barba); aumento do volume dos testículos e tamanho do pênis; oscilação da entonação da voz; alargamento dos ombros e desenvolvimento da massa muscular; início da produção de espermatozoides e início da ejaculação involuntária noturna que acontece durante o sono, caso o menino/homem tenha sonhos eróticos; e nas Meninas - princípio do ciclo menstrual (menarca); alargamento da bacia, podendo desenvolver depósito de gordura nas nádegas, nos quadris e nas coxas; surgimento de pelos nas regiões axilares (axila) e inguinais (pubianos) e desenvolvimento das mamas.

A adolescência é a fase onde ocorre a transição entre a infância e a vida adulta. Nesta fase as mudanças biológicas, psicológicas e sociais são intensas e normalmente acompanhadas por insegurança (MOREIRA BR e FOLMER V, 2015). Para os autores, considerando tantas mudanças nesta fase, os adolescentes precisam do apoio dos adultos de referência (pais, professores e profissionais da saúde) para que suas dúvidas e medos sejam acolhidos. Neste sentido, consideramos que abordar temas acerca da sexualidade com os adolescentes é de extrema relevância e necessidade, haja vista, que o adolescente passa por mudanças biopsicossociais, que podem gerar conflitos com a própria imagem.

Na segunda ação educativa, o tema trabalhado tratou dos Métodos Contraceptivos com a finalidade de esclarecer aos participantes sobre as diversas formas de prevenção das ISTs, bem como, a gravidez não planejada. No primeiro momento, houve uma palestra dinâmica, com exposição dos principais métodos contraceptivos disponíveis. Utilizou-se próteses de silicone para a demonstração da forma correta do uso de preservativos feminino e masculino, diafragma e anel vaginal. Quanto a utilização de materiais didáticos nas atividades extensionistas, Ribeiro et al. (2018) inferem que contribuem efetivamente no processo de apreensão do conhecimento durante as atividades educativas em saúde. No segundo momento realizou-se uma dinâmica de perguntas e respostas, semelhante à primeira intervenção, sendo atingido o objetivo da ação. Ao final os alunos puderam utilizar as próteses de silicone para simular a forma correta de manuseio dos preservativos, sanando eventuais dúvidas.

O conhecimento adequado sobre os diversos métodos contraceptivos disponíveis pelos jovens é de grande importância para que seja feita a melhor escolha, adequando-a às condições socioeconômicas, comportamento sexual, além de auxiliar o uso correto (ALMEIDA LG, et al., 2017). 
Corroborando com que foi observado por Ramos FBP et al. (2019) diz que os adolescentes possuem informações sobre os métodos contraceptivos, principalmente, a camisinha masculina e a pílula anticoncepcional.

A terceira ação abordou o tema IST's, HIV e AIDS. Os discentes executores do projeto confeccionaram uma apresentação em vídeo, sem áudio, com imagens e pequenos textos, relacionados às IST's. Em parceria com a Secretaria de Saúde do Município, foram realizados 100 testes rápidos para Sífilis e HIV. Neste momento todos passaram por um vídeo e orientação pré-teste, em seguida o teste rápido e por fim o resultado foi entregue por um profissional psicólogo da própria Secretaria Municipal onde foi enfatisado as formas de prevenção e como procurar o serviço de saúde caso necessário.Vale salientar que dos testes realizados, tiveram dois resultados positivos para sífilis, onde os jovens foram encaminhados para um Centro de Referência do município.

Ao final da terceira ação ocorreu distribuição de lubrificante íntimo e preservativos femininos e masculinos. O objetivo desta ação foi alcançado, pois existiu participação efetiva do público alvo na realização dos testes, gerando entre os jovens muita curiosidade e interesse. Semelhante ao que foi observado na ação anterior, os estudantes demonstraram ter conhecimento prévio sobre as formas de contágio e prevenção das ISTs, fator positivo no sentido de torná-los menos vulneráveis. Resultados semelhantes foram encontrados no estudo de Costa RSL, et al. (2018). No entanto ainda existe um desconhecimento por parte dos adolescentes sobre os sintomas e as consequências de se adquirir uma IST.

Na quarta ação tratou-se da Gravidez na adolescência. Os discentes do projeto foram atores de uma peça teatral intitulada: "Gravidez na adolescência - A história de Alice", de autoria dos próprios estudantes de Enfermagem, a qual relatava uma situação hipotética de gravidez na adolescência, gerando repercussãos familiares e pessoais. Como dinâmica, durante a exibição da peça teatral, uma adolescente escolar participante do projeto de extensão, recebeu um boneco em forma de um bebê, onde ela deveria simular os cuidados com um recém-nascido, inclusive nos momentos de choro do bebê. Ao final, a adolescente escolar foi convidada a relatar a sua experiência em ter que cuidar do "suposto bebê - seu filho". Em seguida, foi aberto novamente o espaço para que os adolescentes escolares que participavam da ação, pudessem fazer perguntas sobre o tema abordado, onde foi possível observar maior interação dos participantes.

Para os jovens, a adolescência não é o momento ideal para a paternidade, visto que não se consideram com maturidade suficiente para lidar com as demandas desse fenômeno. Essas representações de conotações negativas sugerem que os adolescentes tem consciência de que a gravidez precoce pode ser evitada por meio do adiamento da sexarca, primeira relação sexual, ou mesmo do uso adequado de métodos contraceptivos (BARBOSA AAD, et al., 2016).

Em um estudo realizado por Vieira IB et al. (2017) evidenciou-se que menarca precoce, iniciação da relação sexual antes dos 15 anos, baixa renda familiar, uso inadequado de contraceptivos, ausência de diálogo com os pais, baixo grau de escolaridade e falta de orientações sobre a sexualidade são fatores que predispõem da gravidez na adolescência. Neste sentido é necessário investimentos, por parte dos profissionais de saúde e de educação, na prevenção de uma maternidade/paternidade precoce. Gerando educação em saúde contextualizada e de qualidade que considere as significações dos próprios sujeitos (BARBOSA AAD, et al., 2016).

Na quinta e última ação do Projeto, abordou-se o tema da contaminação pelo Vírus da Imunodeficiência Humana - HIV/AIDS. Foi apresentado um teatro, de autoria própria, cujos atores eram a própria equipe desenvolvedora do projeto, onde era "simulada" a realização dos testes rápidos com jovens escolares. Para cada expectador foi entregue um envelope de papel lacrado na entrada do auditório, o qual continha o resultado de um teste rápido (simulação). No momento em que acontecia a entrega dos resultados foi pedido que cada expectador abrisse o seu e lesse o resultado em silêncio. Durante a intervenção foram exibidos vídeos mostrando "casos reais" de pessoas com o HIV/AIDS. Ao concluir a apresentação da peça, 
os jovens expectadores foram convidados a falar sobre a experiência de receber o resultado, simulado no papel que haviam recebido ao chegar no local da ação. Foi quando se percebeu, mais uma vez, a participação efetiva em falar o que sentiram, se imaginando recebendo o resultado verdadeiro. Ao final da ação ainda houve a distribuição de preservativos feminino e masculino em livre demanda.

Os adolescentes nos últimos anos vem se tornando cada vez mais suscetíveis e expostos a situações de riscos físicos, principalmente à infecção pelo Vírus da Imunodeficiência Humana (HIV), o que afirma a vulnerabilidade desse grupo (THIENGO MA, et al., 2005). Como também observado na pesquisa de Ramos FBP et al. (2019) o desenvolvimento de um projeto de extensão voltado para educação em saúde, leva o aprofundamento dos discentes pesquisadores na temática das IST/HIV/HV e a formação destes como multiplicadores de informações dentro do contexto da população jovem, gerando nos estudantes uma ação de protagonistasno no processo de educação e saúde na perspectiva da população jovem.

\section{CONSIDERAÇÕES FINAIS}

Com o presente trabalho foi possível perceber a ausência de diálogo entre os adolescentes escolares e seus pais e/ou responsáveis, fato que propicia o aumento da vulnerabilidade nesta fase da vida. As ações educativas em saúde transformaram os encontros em momentos de reflexão coletiva, de modo que favoreceram o compartilhamento de experiências, conduzindo a um processo de ensino - aprendizagem participativo. O projeto de extensão também proporcionou aos estudantes do curso Técnico em Enfermagem envolvidos um momento para associar os saberes à prática profissional, por meio da promoção da educação em saúde e da prevenção de doenças desses adolescentes escolares, desenvolvendo conhecimentos e habilidades exigidas durante a formação inicial.

\section{REFERÊNCIAS}

1. ALMEIDA LG, et al. Conhecimento e uso prévio de métodos anticoncepcionais por adolescentes de uma escola pública de Ubá. Revista Científica Fagoc Saúde, 2017; Volume II.

2. AMORAS BC, et al. Reflexões sobre vulnerabilidade dos adolescentes a infecções sexualmente transmissíveis. Revista Eletrônica de Humanidades do Curso de Ciências Sociais da UNIFAP, 2015; 8(1): 163-171.

3. BARBOSA AAD, et al. Representações da gravidez precoce para adolescentes assistidos pela estratégia saúde da família. Revista Norte Mineira de Enfermagem, 2016;5(1):57-73.

4. BIANCO AP. Tema Transversal Orientação Sexual, Prática Pedagógica do professor de Educação Física: Trajetórias e Desafios. Dissertação (Mestrado em Educação Sexual) - Faculdade de Ciências e Letras. Universidade Estadual Paulista (UNESP), Araraquara, 2017; 157 p.

5. BRASIL. Ministério da Saúde. Secretaria de Atenção em Saúde. Departamento de Ações Programáticas Estratégicas. Diretrizes nacionais para a atenção integral à saúde de adolescentes e jovens na promoção, proteção e recuperação da saúde. - Brasília : Ministério da Saúde, 2010.

6. CARNEIRO RF, et al. Educação sexual na adolescência: uma abordagem no contexto escolar. Revista de Políticas Públicas, 2015; 14(1): 104-108.

7. COSTA RSL, et al. Percepção de risco de adolescentes escolares em relação às Infecções Sexualmente Transmissíveis em duas escolas de ensino médio do Acre. DêCiência em Foco, 2018; 2(2): 59-72.

8. KRABBE EC, et al. Escola, sexualidade, práticas sexuais e vulnerabilidades para as Infecções Sexualmente Transmissíveis (IST). Revista Interdisciplinar de Ensino, Pesquisa e Extensão, 2016; 4 (1): 75-84.

9. MOREIRA BR, FOLMER V. Percepções de professores de ciências e educação física acerca da educação sexual na escola. Revista Experiência em Ensino de Ciências, 2015; 10(3): 18-20.

10. OLIVEIRA LP, PATEL BN. Programa de prevenção às infecções sexualmente transmissíveis e HIV/AIDS: uma experiência na Bahia. Revista de extensão Guará, 2015.

11. RAMOS FBP, et. al. A educação em saúde como ferramenta estratégica no desenvolvimento de ações de prevenção da transmissão do HIV: um relato de experiência. Revista Eletrônica Acervo Saúde, 2019; 19(1).

12. RIBEIRO DK, et. al. Experiência Extensionista de Estudantes de Enfermagem em um Projeto de Educação em Saúde e Sexualidade na Escola. Revista Guará, 2018; 9: 85-96.

13. SILVA RAR, et al. Conhecimento de estudantes adolescentes sobre transmissão, prevenção e comportamentos de risco em relação as DST/HIV/AIDS. Rev Fund Care Online, 2016; 8(4): 5054-61.

14. THIENGO MA, et. al. Representações sociais do HIV/AIDS entre adolescentes: implicações para os cuidados de enfermagem. Rev Esc Enferm USP, 2005; 39(1): 68-76.

15. VIEIRA IB, et. al. Fatores de riscos e implicações da gravidez na adolescência: uma revisão integrativa de literatura. Revista Eletrônica Acervo Saúde, 2017; 7(1). 